\title{
Unramified Brauer groups and isoclinism
}

\author{
Primož Moravec \\ Department of Mathematics, University of Ljubljana \\ Jadranska 21, 1000 Ljubljana, Slovenia
}

Received 4 October 2012, accepted 9 April 2013, published online 1 June 2013

\begin{abstract}
We show that the Bogomolov multipliers of isoclinic groups are isomorphic.

Keywords: Unramified Brauer group, Bogomolov multiplier, isoclinism.
\end{abstract}

Math. Subj. Class.: 20F12, 20F35, 13 A50

\section{Introduction}

Let $G$ be a finite group and $V$ a faithful representation of $G$ over an algebraically closed field $k$ of characteristic zero. Suppose that the action of $G$ upon $V$ is generically free. A relaxed version of Noether's problem [11] asks as to whether the fixed field $k(V)^{G}$ is purely transcendental over $k$, i.e., whether the quotient space $V / G$ is rational. A question related to the above mentioned is whether $V / G$ is stably rational, that is, whether there exist independent variables $x_{1}, \ldots, x_{r}$ such that $k(V)^{G}\left(x_{1}, \ldots, x_{r}\right)$ becomes a pure transcendental extension of $k$. This problem has close connection with Lüroth's problem [12] and the inverse Galois problem [14, 13]. By the so-called no-name lemma, stable rationality of $V / G$ does not depend upon the choice of $V$, but only on the group $G$, cf. [4, Theorem 3.3 and Corollary 3.4]. Saltman [13] found examples of groups $G$ of order $p^{9}$ such that $V / G$ is not stably rational over $k$. His main method was application of the unramified cohomology group $\mathrm{H}_{\mathrm{nr}}^{2}\left(k(V)^{G}, \mathbb{Q} / \mathbb{Z}\right)$ as an obstruction. A version of this invariant had been used before by Artin and Mumford [1] who constructed unirational varieties over $k$ that were not rational. Bogomolov [2] proved that $\mathrm{H}_{\mathrm{nr}}^{2}\left(k(V)^{G}, \mathbb{Q} / \mathbb{Z}\right)$ is canonically isomorphic to

$$
\mathrm{B}_{0}(G)=\bigcap_{\substack{A \leq G, A \text { abelian }}} \operatorname{ker}^{G} \operatorname{res}_{A}^{G} \text {, }
$$

where $\operatorname{res}_{A}^{G}: \mathrm{H}^{2}(G, \mathbb{Q} / \mathbb{Z}) \rightarrow \mathrm{H}^{2}(A, \mathbb{Q} / \mathbb{Z})$ is the usual cohomological restriction map. Following Kunyavskiı [7], we say that $\mathrm{B}_{0}(G)$ is the Bogomolov multiplier of $G$.

E-mail address: primoz.moravec@fmf.uni-lj.si (Primož Moravec) 
We recently proved [9] that $\mathrm{B}_{0}(G)$ is naturally isomorphic to $\operatorname{Hom}\left(\tilde{\mathrm{B}}_{0}(G), \mathbb{Q} / \mathbb{Z}\right)$, where $\tilde{\mathrm{B}}_{0}(G)$ is the kernel of the commutator map $G \curlywedge G \rightarrow[G, G]$, and $G \curlywedge G$ is a quotient of the non-abelian exterior square of $G$ (see Section 2 for further details). This description of $\mathrm{B}_{0}(G)$ is purely combinatorial, and allows for efficient computations of $\mathrm{B}_{0}(G)$, and a Hopf formula for $\mathrm{B}_{0}(G)$. We also note here that the group $\tilde{\mathrm{B}}_{0}(G)$ can be defined for any (possibly infinite) group $G$.

Recently, Hoshi, Kang, and Kunyavskiü [6] classified all groups of order $p^{5}$ with nontrivial Bogomolov multiplier; the question was dealt with independently in [10]. It turns out that the only examples of such groups appear within the same isoclinism family, where isoclinism is the notion defined by P. Hall in his seminal paper [5]. The following question was posed in [6]:

Question 1.1 ([6]). Let $G_{1}$ and $G_{2}$ be isoclinic p-groups. Is it true that the fields $k(V)^{G_{1}}$ and $k(V)^{G_{2}}$ are stably isomorphic, or at least, that $\mathrm{B}_{0}\left(G_{1}\right)$ is isomorphic to $\mathrm{B}_{0}\left(G_{2}\right)$ ?

The purpose of this note is to answer the second part of the above question in the affirmative:

Theorem 1.2. Let $G_{1}$ and $G_{2}$ be isoclinic groups. Then $\tilde{\mathrm{B}}_{0}\left(G_{1}\right) \cong \tilde{\mathrm{B}}_{0}\left(G_{2}\right)$. In particular, if $G_{1}$ and $G_{2}$ are finite, then $\mathrm{B}_{0}\left(G_{1}\right)$ is isomorphic to $\mathrm{B}_{0}\left(G_{2}\right)$.

The proof relies on the theory developed in [9]. We note here that we have recently become aware of a paper by Bogomolov and Böhning [3] who fully answer the above question using different techniques. We point out that our approach here is purely combinatorial and does not require cohomological machinery.

\section{Proof of Theorem 1.2}

We first recall the definition of $G \curlywedge G$ from [9]. For $x, y \in G$ we write ${ }^{x} y=x y x^{-1}$ and $[x, y]=x y x^{-1} y^{-1}$. Let $G$ be any group. We form the group $G \curlywedge G$, generated by the symbols $g \curlywedge h$, where $g, h \in G$, subject to the following relations:

$$
\begin{aligned}
g g^{\prime} \curlywedge h & =\left({ }^{g} g^{\prime} \curlywedge{ }^{g} h\right)(g \curlywedge h), \\
g \curlywedge h h^{\prime} & =(g \curlywedge h)\left({ }^{h} g \curlywedge{ }^{h} h^{\prime}\right), \\
x \curlywedge y & =1,
\end{aligned}
$$

for all $g, g^{\prime}, h, h^{\prime} \in G$, and all $x, y \in G$ with $[x, y]=1$. The group $G \curlywedge G$ is a quotient of the non-abelian exterior square $G \wedge G$ of $G$ defined by Miller [8]. There is a surjective homomorphism $\kappa: G \curlywedge G \rightarrow[G, G]$ defined by $\kappa(x \curlywedge y)=[x, y]$ for all $x, y \in G$. Denote $\tilde{\mathrm{B}}_{0}(G)=\operatorname{ker} \kappa$. By [9] we have the following:

Theorem 2.1 ([9]). Let $G$ be a finite group. Then $\mathrm{B}_{0}(G)$ is naturally isomorphic to $\operatorname{Hom}\left(\tilde{\mathrm{B}}_{0}(G), \mathbb{Q} / \mathbb{Z}\right)$, and thus $\mathrm{B}_{0}(G) \cong \tilde{\mathrm{B}}_{0}(G)$.

Let $L$ be a group. A function $\phi: G \times G \rightarrow L$ is called a $\tilde{\mathrm{B}}_{0}$-pairing if for all $g, g^{\prime}, h, h^{\prime} \in G$, and for all $x, y \in G$ with $[x, y]=1$,

$$
\begin{aligned}
\phi\left(g g^{\prime}, h\right) & =\phi\left({ }^{g} g^{\prime},{ }^{g} h\right) \phi(g, h), \\
\phi\left(g, h h^{\prime}\right) & =\phi(g, h) \phi\left({ }^{h} g,{ }^{h} h^{\prime}\right), \\
\phi(x, y) & =1 .
\end{aligned}
$$


Clearly a $\tilde{\mathrm{B}}_{0}$-pairing $\phi$ determines a unique homomorphism of groups $\phi^{*}: G \curlywedge G \rightarrow L$ such that $\phi^{*}(g \curlywedge h)=\phi(g, h)$ for all $g, h \in G$.

We now turn to the proof of Theorem 1.2. Let $G_{1}$ and $G_{2}$ be isoclinic groups, and denote $Z_{1}=Z\left(G_{1}\right), Z_{2}=Z\left(G_{2}\right)$. By definition [5], there exist isomorphisms $\alpha$ : $G_{1} / Z_{1} \rightarrow G_{2} / Z_{2}$ and $\beta:\left[G_{1}, G_{1}\right] \rightarrow\left[G_{2}, G_{2}\right]$ such that whenever $\alpha\left(a_{1} Z_{1}\right)=a_{2} Z_{2}$ and $\alpha\left(b_{1} Z_{1}\right)=b_{2} Z_{2}$, then $\beta\left(\left[a_{1}, b_{1}\right]\right)=\left[a_{2}, b_{2}\right]$ for $a_{1}, b_{1} \in G_{1}$. Define a map $\phi: G_{1} \times G_{1} \rightarrow$ $G_{2} \curlywedge G_{2}$ by $\phi\left(a_{1}, b_{1}\right)=a_{2} \curlywedge b_{2}$, where $a_{i}, b_{i}$ are as above. To see that this is well defined, suppose that $\alpha\left(a_{1} Z_{1}\right)=a_{2} Z_{2}=\overline{a_{2}} Z_{2}$ and $\alpha\left(b_{1} Z_{1}\right)=b_{2} Z_{2}=\overline{b_{2}} Z_{2}$. Then we can write $\overline{a_{2}}=a_{2} z$ and $\overline{b_{2}}=b_{2} w$ for some $w, z \in Z_{2}$. By the definition of $G_{2} \curlywedge G_{2}$ we have that $\overline{a_{2}} \curlywedge \overline{b_{2}}=a_{2} z \curlywedge b_{2} w=a_{2} \curlywedge b_{2}$, hence $\phi$ is well defined.

Suppose that $a_{1}, b_{1} \in G_{1}$ commute, and let $a_{2}, b_{2} \in G_{2}$ be as above. By definition, $\left[a_{2}, b_{2}\right]=\beta\left(\left[a_{1}, b_{1}\right]\right)=1$, hence $a_{2} \curlywedge b_{2}=1$. This, and the relations of $G_{2} \curlywedge G_{2}$, ensure that $\phi$ is a $\tilde{\mathrm{B}}_{0}$-pairing. Thus $\phi$ induces a homomorphism $\gamma: G_{1} \curlywedge G_{1} \rightarrow G_{2} \curlywedge G_{2}$ such that $\gamma\left(a_{1} \curlywedge b_{1}\right)=a_{2} \curlywedge b_{2}$ for all $a_{1}, b_{1} \in G_{1}$. By symmetry there exists a homomorphism $\delta: G_{2} \curlywedge G_{2} \rightarrow G_{1} \curlywedge G_{1}$ defined via $\alpha^{-1}$. It is straightforward to see that $\delta$ is the inverse of $\gamma$, hence $\gamma$ is an isomorphism.

Let $\kappa_{1}: G_{1} \curlywedge G_{1} \rightarrow\left[G_{1}, G_{1}\right]$ and $\kappa_{2}: G_{2} \curlywedge G_{2} \rightarrow\left[G_{2}, G_{2}\right]$ be the commutator maps. Since $\beta \kappa_{1}\left(a_{1} \curlywedge b_{1}\right)=\beta\left(\left[a_{1}, b_{1}\right]\right)=\left[a_{2}, b_{2}\right]=\kappa_{2} \gamma\left(a_{1} \curlywedge b_{1}\right)$, we have the following commutative diagram with exact rows:

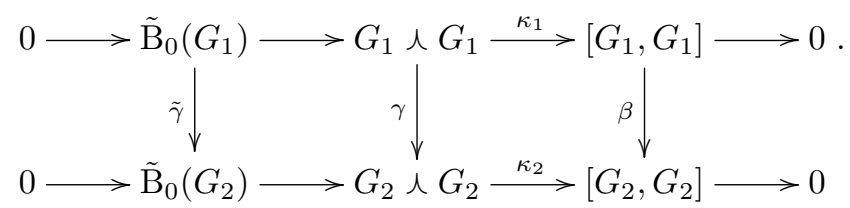

Here $\tilde{\gamma}$ is the restriction of $\gamma$ to $\tilde{\mathrm{B}}_{0}\left(G_{1}\right)$. Since $\beta$ and $\gamma$ are isomorphisms, so is $\tilde{\gamma}$. This concludes the proof.

\section{References}

[1] M. Artin and D. Mumford, Some elementary examples of unirational varieties which are not rational, Proc. London. Math. Soc. 25 (1972), 75-95.

[2] F. A. Bogomolov, The Brauer group of quotient spaces by linear group actions, Izv. Akad. Nauk SSSR Ser. Mat. 51 (1987), 485-516.

[3] F. Bogomolov and C. Böhning, Isoclinism and stable cohomology of wreath products, arXiv:1204.4747v1.

[4] J.-L. Colliot-Thélène and J.-J. Sansuc, The rationality problem for fields of invariants under linear algebraic groups (with special regards to the rationality problem), in: V. Mehta (ed.), Proceedings of the International Colloquium on Algebraic groups and Homogeneous Spaces (Mumbai 2004), TIFR, Mumbai, Narosa Publishing House (2007), 113-186.

[5] P. Hall, The classification of prime-power groups, J. Reine Angew. Math. 182 (1940), 130-141.

[6] A. Hoshi, M. Kang and B. E. Kunyavskiǔ, Noether problem and unramified Brauer groups, ArXiv:1202.5812v1, 2012.

[7] B. E. Kunyavskiı̌, The Bogomolov multiplier of finite simple groups, Cohomological and geometric approaches to rationality problems, 209-217, Progr. Math., 282, Birkhäuser Boston, Inc., Boston, MA, 2010. 
[8] C. Miller, The second homology of a group, Proc. Amer. Math. Soc. 3 (1952), 588-595.

[9] P. Moravec, Unramified groups of finite and infinite groups, Amer. J. Math., to appear, arXiv:1203.3190v1.

[10] P. Moravec, Groups of order $p^{5}$ and their unramified Brauer groups, J. Algebra, to appear, arXiv:1203.3289v1.

[11] E. Noether, Gleichungen mit vorgeschriebener Gruppe, Math. Ann. 78 (1916), 221-229.

[12] I. R. Šafarevič, The Lüroth problem, Proc. Steklov Inst. Math. 183 (1991), 241-246.

[13] D. J. Saltman, Noether's problem over an algebraically closed field, Invent. Math. 77 (1984), 71-84.

[14] R. G. Swan, Noether's problem in Galois theory, in 'Emmy Noether in Bryn Mawr', SpringerVerlag, Berlin, 1983. 